\title{
Cranial Ultrasonography and Doppler in Preterm and Term Neonates
}

\author{
Satish Prasad B.S ${ }^{1}$, Sreenivasa Raju N ${ }^{2}$, Surabi Chakraborty ${ }^{3}$ \\ 1. Professor and HOD, Department of Radiodiagnosis, Adichunchangiri Institute Of Medical Sciences , mandya \\ dist, Karnataka state, India \\ 2. Junior Resident, Department of Radiodiagnosis, Adichunchangiri Institute Of Medical Sciences, mandya dist \\ ,Karnataka state, India. \\ 3. Junior Resident, Department of Radiodiagnosis, Adichunchangiri Institute Of Medical Sciences, mandya dist \\ , Karnataka state, India..
}

\begin{abstract}
:
Aims And Objective: Role of cranial ultrasonography to evaluate intracranial abnormalities in preterm and term neonates.

$>$ To ascertain the clinical course of intracranial haemorrhage and to determine the short-term outcome of the affected baby neonates who develop intracranial complications of prematurity.

$>$ The utility of real-time ultrasonography in the diagnosis of neonatal periventricular leukomalacia.

To asses utility of cranial colour Doppler in preterm and term neonates.

$>$ To evaluate cerebral blood flow velocities in ACA, MCA and PCA in preterm and term neonates.

$>$ To evaluate developmental interval changes in cerebral postnatal hemodynamic variation in the blood flow velocities in the preterm and term neonates.

Method: Grey scale imaging for intracranial abnormalities and colour Doppler evaluation of intracranial arteries were done on neonates between 3 to 5 days, 10 to 14 days and 26 to 30 days after birth. RESULT: The study was conducted in the department of radiodiagnosis in Adichunchangiri Institute Of Medical Sciences. Prospective radiological clinical study with 52 neonates was undertaken to study the Cranial ultrasonography and Doppler in Preterm $(<37$ weeks) and Term $(>37$ weeks).On cranial USG , 15 (28.8\%) neonates revealed no abnormality, 12(23.1\%) showed PVHE , 11(21.2\%)neonates showed ICH, 6(11.5\%) neonates showed PVL, 4(7.7\%) neonates showed Cysts, 4(7.7\%) neonates had cerebral edema. On cranial Doppler evaluation of ACA, MCA and PCA progressive rise in systolic and diastolic velocities were seen with increasing gestational age and increasing age of the neonate. The rate of increase in velocity was greater with increasing gestational age. The resistive index decreased progressively with gestational age and with increasing age of the neonate.

Conclusion: Neonatal cranial ultrasonography is the easiest and most ideal investigation for diagnosing intracranial pathologies in preterm and term neonates. Neonatal cranial Doppler provides a reliable means to evaluate changes in the intracerebral blood flow with a variety of intracranial pathologies.
\end{abstract}

Key Words: Cranial USG, Cranial Doppler, neonates.

\section{Introduction}

The newborn brain is vulnerable to injury from many causes, like preterm delivery, hypoxia, trauma etc, resulting in significant mortality and morbidity despite recent improvements in the neonatal intensive care. ${ }^{1}$ Cranial USG are widely used to identify preterm neonates at risk for brain injury and subsequent neurodevelopmenal defects, most commonly as a consequence of severe intraventricular haemorrhage (IVH) and cystic periventricular leukomalacia (PVL). ${ }^{1-3}$ Cerebroventricular haemorrhage , the most common lesion ,originates in the germinal matrix, a structure located beneath the ependymal lining of the ventricles and in th groove between the head of the caudate nucleus and the thalamus. It is a highly vascular structure with little supporting tissue. It is a source of neuroblasts which migrate peripherally during the development of the fetal brain. The germinal matrix is largest at 24-32 weeks gestation and then involutes so that it is much smaller in full term infants than preterm. Intraventricular haemorrhage occurs in $40 \%$ of preterm neonates who weigh less than $1500 \mathrm{~g} ; 90 \%$ of haemorrhages occur within first 3 postnatal days and the remainder by 10 days. ${ }^{3-6}$ Intraventricular haemorrhage is usually clinically occult and detection requires a screening USG. ${ }^{3}$

Cranial USG in neonates almost always reveals a hyperchogenicity just posterior and superior to the ventricular trigone on parasagittal view. The peritrigonal echogenic blush, appearing on parasagittal sonograms as a grouping of fine, linear densities almost like brush strokes is virtually always present on cranial sonograms of premature neonates. Periventricular leukomalacia is the second most frequent lesion of the infant brain, following haemorrhage into the germinal matrix or ventricle. PVL is now considered the principal form of brain 
injury among preterm infants. PVL is the major reason for developing a variety of neurological sequelae ; including motor dysfunction; delayed cognitive development ; visual impairment and epilepsy seen in $3 \%$ to $10 \%$ of premature neonates. ${ }^{2}$ The sonographic criteria of PVL are increased white matter echogenicity that evolves to cyst formation. ${ }^{3}$ However, clinically significant white matter injury may occur without frank cyst formation and in the absence of white matter changes on USG, as evidenced by development of non obstructive ventriculomegaly not associated with IVH. Cystic PVL is invariably associate with significant long-term neurologic morbidity, early identification of preterm infant at highest risk for the subsequent development of this lesion is critical. Early identification may facilitate future preventive strategies.

In the recent years, the incidence of all grades of haemorrhage has decreased because of increased antenatal steroid use and improved neonatal care. ${ }^{8,9}$ As cost containment has become a more pressing issue, the need for and frequency of screening USG has been raised. ${ }^{10-12}$ Spontaneous haemorrhage in and around the cerebral ventricle is a phenomenon that occurs in premature neonates ${ }^{13}$ and is now being increasingly observed in the high risk term neonates. ${ }^{14}$ It's incidence is approximately $40-45 \%$ in newborns weighing less than $500 \mathrm{~g}$ or born before 35 weeks of gestational age. It is due to rupture of fragile capillaries of the germinal matrix whereas hypoperfusion may cause infarction of the boundary zones between different arterial territories, within the periventricular white matter.

Conventional colour Doppler sonography has been the main sDoppler technique used in conjunction with grey-scale evaluation of the neonatal brain for the assessment of parenchymal vascularity. Conventional colour Doppler sonography measures frequency shifts and direction of blood flow.

Transcranial Doppler sonography is emerging technique. The procedure is non invasive, ionising, portable, safe for serial or prolonged studies and performed with relatively inexpensive equipment. Doppler transducer used measure the velocity of blood flow within the circle of Willis. By spectral analysis of frequency shifts from insonated red blood cells moving through a preselected arterial sample volume, transcranial Doppler calculate and displays the peak systolic velocity(PSV) and end diastolic velocity(EDV), the mean velocity and the resistive index(RI) of blood flow within the interrogated vessel. ${ }^{17}$

\section{Materials And Methods}

This is a prospective study conducted over a period of 2 years. A total of 52 neonates were evaluated during the study period. The examinations were done between 3-5 days, $10-14$ days and 26- 30 days after birth. Every effort was taken to maintain normal body temperature in preterm infants by use of overhead warming lamps, blankets and warmed coupling gel. Hand washing and cleaning of the transducers with a disinfectant are of paramount importance to avoid the spread of infection in the intensive care unit. INCLUSION CRITERIA: All neonates born within 28 to 42 weeks gestational age. The study included screening as well as clinically indicated scans and included cases of ICH, PVL, diffuse cerebral edema, solitary cysts, transient periventricular hyperchogenicity and 15 normal cases. The birth weight and gestational age (as assessed using the modified Ballard measure) were also recorded. ${ }^{18}$ EXCLUSION CRITERIA: Neonates with congenital heart disease and any other heart disease, the presence of which may alter cerebral flow velocities. Neonates which were incompletely examined due to early discharge and died during the study period. The USG machine "Voluson S6 Pro" from GE with a high resolution array linear transducer of 7-10 MHz was used during the study. Standard coronal, sagittal and parasagittal imaging were carried out using anterior fontanelle as acoustic window to evaluate parenchymal , ventricular, subarachnoid space abnormalities and any other malformations. ${ }^{17}$ Sonographic examination was carried out using Transtemporal approach, the sonographic image obtained being similar in detail to that seen on an anatomic slice at $10^{\circ}$ through the suprasellar cistern. The vessels seen as pulsating structures , ACA curving anteriorly , the MCA in the sylvian fissure and PCA lying between the uncus of the temporal lobe and superior cerebral peduncle. ${ }^{16}$ Spectral tracings were obtained from the respective arteries with Doppler angle $<60^{\circ}$. The Doppler indices PSV, EDV and RI were measured in a representative waveform in each patient. ${ }^{16}$ Descriptive statistical analysis has been carried out in the present study. The statistical software namely SPS 15., Stata 8.0, Med Calc 9.0.1 and Systat 11.0 were used for the analysis of the data.

\section{Results}

This study included a cohort of total 52 neonates who were admitted in NICU of which 33 were preterm and 19 were term (Table 3 and 4). The mean gestational age of neonate was $35.5 \pm 3.08$ weeks (Table 2). The mean birth weight of neonate was $2251.73 \pm 395.39 \mathrm{gm}$ (Table5).

FLARING: Total of 12 neonates (23.1\%) were having flaring, all the neonates were preterm (36.4\%) (Table 3) and weighing between 2001 to $2500 \mathrm{gm}$ (54.6\%) (Table 7). This flaring was present between 3 to 5 days scan (Table 9) which subsequently became normal between 10-14 days and 26-30 days scans(Table 10 and 11).

INTRACRANIAL HAEMORRHAGE: 11 neonates $(21.2 \%)$ (Table 1) were having ICH, of which 10 neonates were preterm $30.3 \%$ (Table 3 ) and one neonate was term $5.3 \%$ (Table 4 ). Neonates weighing $<2000 \mathrm{gm}$, 
6 had ICH type I (42.9\%) and 1 had ICH type II (7.1\%)(Table 6). Neonates weighing between 2001 to $2500 \mathrm{gm}$, 4 had ICH type 1 (18.2\%) (Table 7). Cranial USG done between 3-5 days scan revealed 10 ICH type I (19.2\%) and 1 ICH type II (1.9\%)(Table 9). On subsequent Cranial USG done between 10 to 14 days scan revealed 6 ICH type I $(11.5 \%)$ as reducing and $3 \mathrm{ICH}$ type I( $5.8 \%), 1 \mathrm{ICH}$ type II (1.9\%) as same (Table 10). Cranial USG done between 26 to 30 days scan revealed 2 ICH type $1(3.9 \%), 1$ ICH type II $(1.9 \%)$ as reducing and 1 ICH type $(1.9 \%)$ as same (Table 11$)$.

PVL: 6 neonates (11.5\%)(Table 1) were having PVL; all the neonates were preterm (18.2\%)(Table 3) and weighing less than 2000gm (42.9\%)(Table 6). Cranial USG done between 3 to 5 days scan revealed 6 flaring (11.5\%)(Table 9). On subsequent Cranial USG done between !0 to 14 days scan revealed (11.5\%)(Table 10). Cranial USG done between 26 to 30days scan revealed 6 PVL (11.5\%)(Table 11).

CYSTS: Total 4 neonates (7.7\%)(Table 1) were having cysts, 1 neonate was preterm (3.0\%)(Table3) and 3 neonates were term $(15.8 \%)$ (Table 4). Neonates weighing between 2001 to $2500 \mathrm{gm}$ had 2 cysts $(9.1 \%)$ (Table 7). Neonates weighing $\geq 2501,2$ had cysts( $(12.5 \%)$ (Table 8). Cranial USG done between $3-5$ days san revealed 4 cysts $(7.7 \%)$ (Table 9). On subsequent scans between 10 to 14 days and 26 to 30 days showed persistence of these cysts $7.7 \%$ (Table 10 ) and $7.7 \%$ (Table 11 ) respectively.

CEREBRAL EDEMA: Total 4 neonates $(7.7 \%)($ Table 1) were having cerebral edema; all were preterm $(12.1 \%)$ (Table 3). 1 neonate weighing $<2000 \mathrm{gm} 7.1 \%$ (Table 6 ) and the remaining three neonates were weighing between 2001 to $2500 \mathrm{gm}(13.6 \%$ )(Table 7).Cranial USG done between 3-5 days scan revealed 4 cerebral edema $7.7 \%$ (Table 9). On subsequent scans done between 10 to 14 days showed reduction in cerebral edema $7.7 \%$ (Table 10). Cranial USG done between 26 to 30 days showed complete resolution of cerebral edema and became normal.

Cranial color Doppler : Analysis of data over 3 to 5 days , 10 to 14 days and 26 to 30 days shows there is a progressive rise in systolic and diastolic velocities regardless of the initial gestational age. However, the higher the initial gestational age the greater the rate of increase in velocity. With increasing gestational age the resistive index is seen to fall. (Table 12).

IV. Tables:

\begin{tabular}{|l|c|c|}
\hline \multicolumn{1}{|c|}{ Cranial USG findings } & Number of cases & \% \\
\hline Normal & 15 & 28.8 \\
\hline Flaring/ PVHE & 12 & 23.1 \\
\hline ICH & 11 & 21.2 \\
\hline PVL & 6 & 11.5 \\
\hline Cyst & 4 & 7.7 \\
\hline Cerebral edema & 4 & 7.7 \\
\hline Total & 52 & 100.0 \\
\hline \multicolumn{2}{|c|}{ Table 1: Grand total distribution of number of cases in Preterm and Term neonates. } \\
\hline
\end{tabular}

Table 1 shows grand total distribution of number of cases in Preterm and Term neonates.

\begin{tabular}{|l|c|c|}
\hline Gestational age (weeks) & Number of cases & \% \\
\hline $28-34$ & 19 & 36.5 \\
\hline $35-36$ & 14 & 27.0 \\
\hline $37-39$ & 13 & 25.0 \\
\hline $40-42$ & 6 & 11.5 \\
\hline Total & 52 & 100.0 \\
\hline Table 2: Gestational age distribution of neonates studied & & \\
\hline
\end{tabular}

Table 2 shows gestational age distribution of neonates studied.

\begin{tabular}{|l|c|c|}
\hline \multicolumn{1}{|c|}{ Cranial USG findings in Preterm } & Number of cases & \% \\
\hline Flaring/ PVHE & 12 & 36.4 \\
\hline ICH & 10 & 30.3 \\
\hline PVL & 6 & 18.2 \\
\hline Cyst & 4 & 12.1 \\
\hline Cerebral edema & 1 & 3.0 \\
\hline Total & 33 & 100.0 \\
\hline Table 3: Distribution of number of cases in Preterm neonates.
\end{tabular}

Table 3 shows distribution of number of cases in Preterm neonates.

\begin{tabular}{|l|c|c|}
\hline \multicolumn{1}{|c|}{ Cranial USG findings in Term } & Number of cases & \% \\
\hline Normal & 15 & 78.9 \\
\hline ICH & 3 & 15.8 \\
\hline Cyst & 1 & 5.3 \\
\hline Total & 19 & 100.0 \\
\hline Table 4: Distribution of number of cases in Term neonates.
\end{tabular}

Table 4 shows distribution of number of cases in Term neonates. 


\begin{tabular}{|l|c|c|}
\hline \multicolumn{1}{|c|}{ Birth weight (grams) } & Number of cases & \% \\
\hline$<2000$ & 14 & 26.9 \\
\hline $2001-2500$ & 22 & 42.3 \\
\hline $2501 \&$ above & 16 & 30.8 \\
\hline Total & 52 & 100.0 \\
\hline Table 5: Birth weight of neonate studied & & \\
\hline
\end{tabular}

Table 5 shows birth weight of neonate studied.

\begin{tabular}{|l|c|c|}
\hline \multicolumn{1}{|c|}{ Cranial USG findings in Term } & Number of cases & \% \\
\hline PVL & 6 & 42.9 \\
\hline ICH type I & 6 & 42.9 \\
\hline ICH type II & 1 & 7.1 \\
\hline Cerebral edema & 1 & 7.7 \\
\hline Total & 14 & 100.0 \\
\hline Table 6: Cranial USG findings in neonates less than 2000 grams
\end{tabular}

Table 6 shows cranial USG findings in neonates less than 2000 grams.

\begin{tabular}{|l|c|c|}
\hline \multicolumn{1}{|c|}{ Cranial USG findings } & Number of cases & \% \\
\hline Flaring/ PVHE & 12 & 54.6 \\
\hline ICH type I & 4 & 18.2 \\
\hline Cerebral edema & 3 & 13.6 \\
\hline Cyst & 2 & 9.1 \\
\hline Normal & 1 & 4.5 \\
\hline Total & 22 & 100.0 \\
\hline Table 7: Cranial USG findings in neonates between 2001-2500 grams & \\
\hline
\end{tabular}

Table 7 shows Cranial USG findings in neonates between 2001-2500 grams

\begin{tabular}{|l|c|c|}
\hline \multicolumn{1}{|c|}{ Cranial USG findings } & Number of cases & \% \\
\hline Normal & 14 & 87.5 \\
\hline Cyst & 2 & 12.5 \\
\hline Total & 16 & 100.0 \\
\hline Table 8: Cranial USG findings in neonates, 2501 gram and above.
\end{tabular}

Table 8 shows cranial USG findings in neonates , 2501 gram and above.

\begin{tabular}{|c|c|c|}
\hline Cranial USG findings in Term & Number of cases & $\%$ \\
\hline Normal & 15 & 28.9 \\
\hline Flaring/PVHE & 18 & 34.6 \\
\hline ICH type I & 10 & 19.2 \\
\hline ICH type II & 1 & 1.9 \\
\hline Cyst & 4 & 7.7 \\
\hline Cerebral edema & 4 & 7.7 \\
\hline Total & 52 & 100.0 \\
\hline
\end{tabular}

Table 9 shows cranial USG findings in neonates done between 3 to 5 days.

\begin{tabular}{|c|c|c|}
\hline Cranial USG findings in Term & Number of cases & $\%$ \\
\hline Normal & 28 & 53.9 \\
\hline Reducing & 10 & 19.2 \\
\hline ICH type I & 6 & 11.5 \\
\hline Cerebral Edema & 4 & 7.7 \\
\hline Same & 14 & 26.9 \\
\hline ICH Type I & 3 & 5.8 \\
\hline ICH type II & 1 & 1.9 \\
\hline PVHE/flaring & 6 & 11.5 \\
\hline Cyst & 4 & 7.7 \\
\hline Total & 52 & 100.0 \\
\hline
\end{tabular}

Table 10 shows Cranial USG findings in Term done between 10 to 14 days.

\begin{tabular}{|l|c|c|}
\hline Cranial USG findings & Number of cases & \% \\
\hline Normal & 38 & 73.1 \\
\hline PVL & 6 & 11.5 \\
\hline Reducing & 3 & 5.8 \\
\hline ICH type I & 2 & 3.9 \\
\hline ICH type II & 1 & 1.9 \\
\hline Same & 5 & 9.6 \\
\hline ICH Type I & 1 & 1.9 \\
\hline Cyst & 4 & 7.7 \\
\hline
\end{tabular}



52

Table 11 shows cranial USG findings done between 26 to 30 days.

\begin{tabular}{|c|c|c|c|c|}
\hline Pair & $\begin{array}{c}\text { Findings } \\
\end{array}$ & 3 to 5 days & 10 to 14 days & 26 to 30 days \\
\hline \multirow{3}{*}{ GA vs ACA } & GA vs PSV $(\mathrm{cm} / \mathrm{sec})$ & $0.596 * *$ & $0.610^{* * *}$ & $0.629 * *$ \\
\hline & GA vs EDV $(\mathrm{cm} / \mathrm{sec})$ & $0.602 * *$ & $0.593 * *$ & $0.592 * *$ \\
\hline & GA vs RI & $-0.591 * *$ & $-0.513^{* *}$ & $-0.469 * *$ \\
\hline \multirow{3}{*}{ GA vs MCA } & GA vs PSV $(\mathrm{cm} / \mathrm{sec})$ & $0.601 * *$ & $0.601 * *$ & $0.610 * *$ \\
\hline & GA vs $\operatorname{EDV}(\mathrm{cm} / \mathrm{sec})$ & $0.607 * *$ & $0.625 * *$ & $0.628 * *$ \\
\hline & GA vs RI & $-0.602 * *$ & $-0.610 * *$ & $-0.587 * *$ \\
\hline \multirow{3}{*}{ GA vs PCA } & GA vs PSV $(\mathrm{cm} / \mathrm{sec})$ & $0.601 * *$ & $0.611 * *$ & $0.618^{* *}$ \\
\hline & GA vs $\operatorname{EDV}(\mathrm{cm} / \mathrm{sec})$ & $0.609 * *$ & $0.618 * *$ & $0.616 * *$ \\
\hline & GA vs RI & $-0.590 * *$ & $0.644 * *$ & $-0.614 * *$ \\
\hline
\end{tabular}

Table 12 shows correlation between GA and cranial Doppler evaluation.

**, Highly statistical significant at $\mathrm{p}<0.001$
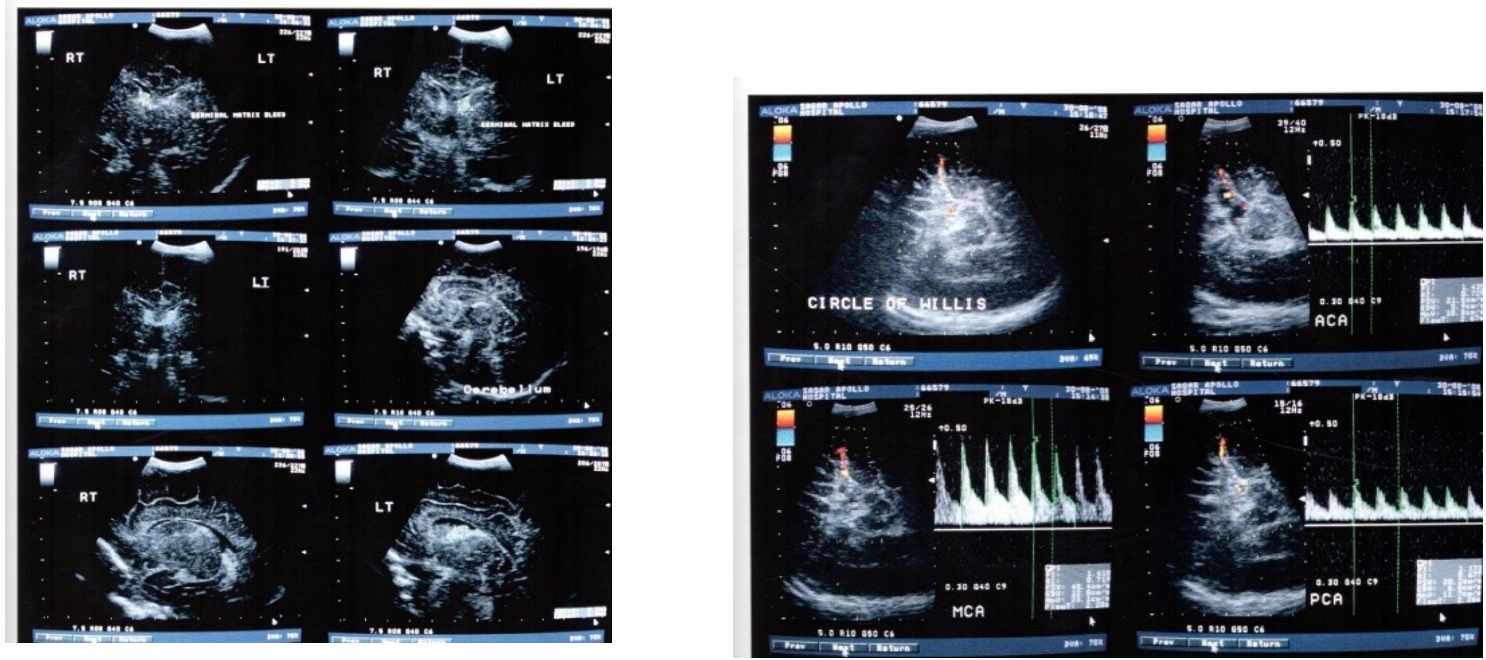

34 weeks preterm neonate weighing 2215 grams with clinical history of hyperbilirubinemia, systemic candidiasis. Cranial USG was done on 3 to 5 days showing bilateral, grade I germinal matrix bleed (ICH type I ) in caudo-thalamic grooves. The bleeds are projecting in to the ventricles with out intraventricular extension. On follow up scan done on 10 to 14 days the bleed was reducing, and on 26 to 30 days it became normal.

Cranial Doppler evaluation of same baby done on 3 to 5 days.
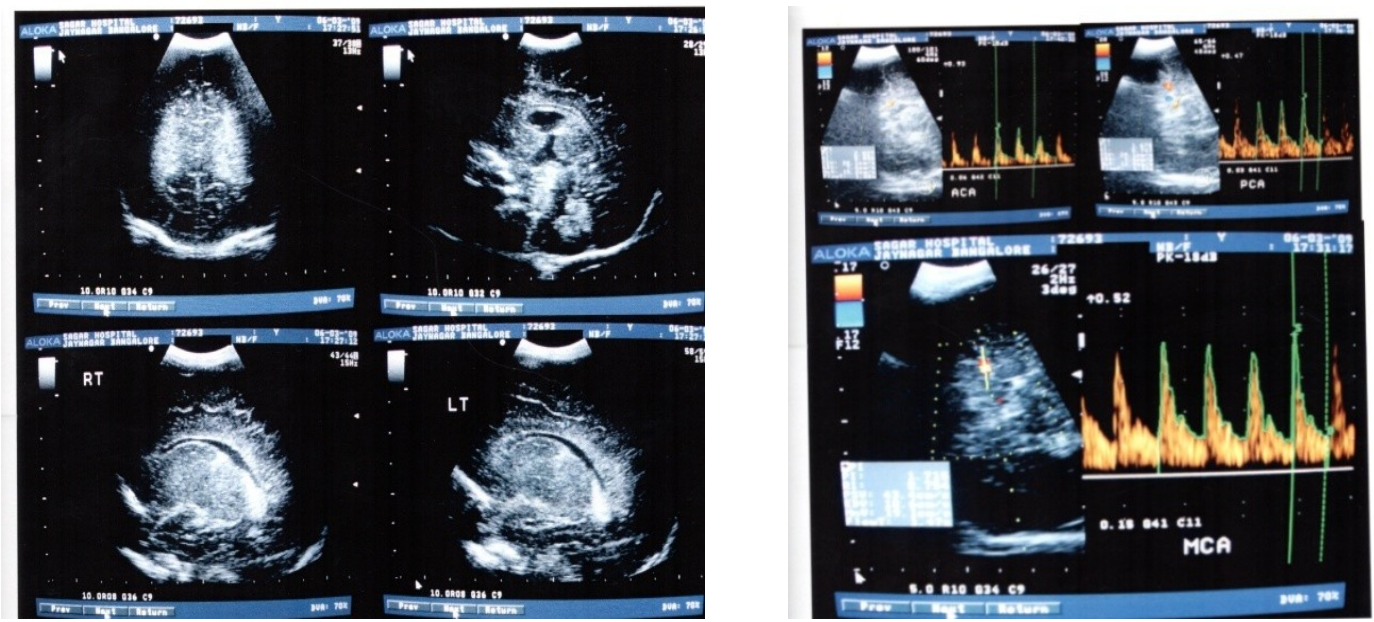

35 weeks preterm neonate weighing 2180 grams with clinical history of asphyxia, sepsis, hyperbilirubinemia.

Cranial USG was done on 3 to 5 days showing bilateral periventricular hyperechogenecity (PVHE) / flaring. On follow up scans done on 10 to 14 days, and on 26 to 30 days it became normal.

Cranial Doppler evaluation of same baby done on 3 to 5 days. 


\section{Discussion}

Cranial ultrasonography is a well established diagnostic technique used in the evaluation of intracranial anatomy and pathology in neonate. Cranial USG had diagnosed intracranial haemorrhage(ICH), subependymal haemorrhage (SEH), periventricular leukomalacia , cerebral edema and cysts.

Because there is generally a poor concordance between clinical status and intracranial pathology in the highrisk premature neonate, serial neurosonography was performed in our neonatal intensive care unit. Although $\mathrm{ICH}$ and PVL were the most common abnormalities seen, 4 neonates demonstrated cysts and 4 neonates demonstrated cerebral edema.

Color Doppler sonography to assess intracranial blood flow was done on 52 neonates which included both preterm and term. The anterior, middle and posterior cerebral arteries were evaluated by temporal approach. Intracranial velocities were seen to increase with increasing gestational age and increasing age of the neonate; the resistive index decreased progressively with age. Thus, the resistive index exhibits the opposite trend to the true velocity movement. The reason for the falling resistive index is due to the relative increase occurring in systolic and diastolic velocities with time. The rate of increase in velocity was greater with higher gestational age. This concern is further emphasized that blood flow in the neonatal brain also increases with gestational age and with age of neonate. MCA velocities were greater than ACA and PCA velocities.

The mechanism of autoregulation perhaps offer clues in the investigation of intracranial haemorrhage, which may be caused by sudden changes in the blood flow velocity. A relatively low-velocity differential between systole and diastole in premature neonates indicates a relatively dilated intravascular bed and suggests these neonates may be less able than term neonates to withstand transient vascular disturbances, which may be the basis for intracranial haemorrhage and ischemia.

\section{Conclusion}

Cranial USG uses are a technique that capitalizes on the bone free anterior fontanelle to provide a kind of window into the neonatal brain. It is the easiest and most ideal investigation for diagnosing intracranial pathologies in preterm and term neonates, which is sensitive for detection of ICH, PVL and hydrocephalus. Cranial colour Doppler now provides a powerful tool to evaluate the neonatal intracerebral blood flow in a noninvasive manner. The transtemporal approach provides a reliable means of evaluating MCA, ACA and PCA. The current emphasis on standardisation of examinations and interpretation will improve the accuracy of sonography for making diagnosis and affect patients outcome positively. We must look at what we need to know to care for a baby as opposed to what we want to know to satisfy our curiosity. Outcome-based research on the use of cranial sonography of neonate should be encouraged and supported.

\section{References:}

[1]. Perlman JM , Rollins N, Burns D, Risser R. Relationship periventricular intraparenchymal echogenicitiy and germinal matrixintraventricular haemorrhage in the very low birth weight infant. Pediatrics. 1991; 91: 474-480.

[2]. Perlman JM, Risser R, Broyles RS . Bilateral cystic periventricular leukomalacia in the premature infant: associated risk factors . Pediatrics. 1996; 97:822-827.

[3]. Vople JJ. Neurology of the Newborn. $3^{\text {rd }}$ ed. Philadelphia, Pa: WB Saunders Co; 1995: 403-467.

[4]. Levene MI, Starte DR. A longitudinal study of post-hemorrhagic ventricular dilatation in the newborn. Arch Dis Child . 1981; 56: $905-908$.

[5]. Ahmann PA, Lazzarra A, Dykes FD, Brann AW Jr, Schwartz JF. Intraventricualr haemorrhage in the high risk preterm infant : incidence and outcome. Ann Neurol. 1980; 118-124.

[6]. Prelman JM, Volpe JJ . Intraventricular hemorrhage in extremely small premature infants . AJDC. 1986; 140:122-124.

[7]. Stewart AL , Reynolds EO, Hope RL, et al. Probability of neurodevelopmental disorders estimated fron ultrasound appearances of brain of very preterm infants, Dev Med child Neurol. 1987; 29: 3-11.

[8]. Wright LL, Home JD, Gunkel H. Evidence from multicentre networks on the current use and effectiveness of antenatal corticosteroids in low birth weight infants . AM=m J Obstet Gynecol. 1995; 173: 263-269.

[9]. Philips Ag, Allen WC, Tito AM, Wheeler LR. Intraventricular haemorrhage in preterm infants : declining evidence in the 1980s, Pediatrics 1989; 84: 797-801.

[10]. Boal DK, Walterberg KL, Niles S, Gifford KL. Optimal cost-effective timing of cranial ultrasound screening in low birth weight infants. Pediatr Radiol. 1995; 25: 425-428.

[11]. Chess PR, Chess MA , Guillet R. Screening head ultrasound to detect intraventricular haemorrhage in premature infants. Pediar Radiol. 1997; 27: 305-308

[12]. Paul DA, Pearlman SA, Finkelstein MS, Stefano JL. Cranial ultrasound in very low birth weight infants : do all inants need to be screened? Clin Pediatr. 1999; 38: 503-509.

[13]. Leech RW, Kohnen P. Subependymal and intraventricular haemorrhages in the newborn. Am J Pathol 1974: 77;465-476.

[14]. Palma PA, Miner ME, Morris FH Jr., Adcock EW, Densen SE. Intraventricular haemorrhage in the neonate born at term. AM J Dis Child1979,133: 941-944.

[15]. Anthony R. Lupetin, Donalee A. Davis, CNRN Irwin Beckman, Nilima Dash. Transcranial Doppler Sonongraphy Part 1. Principles , Technique and Normal Appearances. Radiographics 1995; 15:109-119.

[16]. Gerard Horgan J, Carol M. Rumack, Thomas Hay, Michael L. Manco - Johnson, Geral B. Merenstein ,Chris Esola. Absolute Intracranial Blood Flow Velocities Evaluated by duplex Sonography in asymptomatic preterm and term neonates. AJR 1989 May; $152: 1059-1064$.

[17]. Jeffrey M. Perlman and Nancy Rollins . Surveillance Protocol for the Detection of Intracranial Abnormalities In Preterm Neonates. Arch Pediatr Adolesc Med. 2000;154: 822-826.

[18]. Davis PJC, Cox RM and Brooks J. Training in neonatal cranial ultrasound: a questionnaire survey . BJR 2005; 78: 55-56. 